\title{
Publications Received for Review
}

Phonetica 1996:53:115-116

Each fascicle of Phonerica provides a list of the publications that have been received for review. Readers who would like (1) write critical commentarics on any of these books for Phonetica are requested to contact the editor, Prof. K. J. Kohler Institut für Phonetik, Universität Kicl, Olshausenstr. 4(), D-24()98 Kiel. FRG, who will arrange for the copies to be sent to them. At the same time. he will inform them about the expected length of the review as well as other technical details, and suggest a date for submitting the manuscripts. The time allotted for preparing reviews will on principle be kept as siont as possible in order to enable Phonetica (o) fulfill its obligation of kecping its readers up to date with publications in the field of speech science. Readers are also welcome to suggest any other book in our field for review in Phonetica. over and above the ones named in the list of received publications. The editor will then take the necessary steps to obtain copies from the publishing firms.

Ahercombie. David: Fifty Years in Phonetics. Selected Papers. Edinburgh University Press, Edinburgh 1991. 127 pp., \$25.00): ISBN (0) $7486(01961$.

Aleer: Peter: Phonologie der Alltagssprache. Fine Untersuchung zur Standard/Dialcktvariation am Beispiel der Konstanzer Stadtsprache (= Studia Linguistica Germanical 28). Walter de Gruyter. Berlin. New York 199(). DM 164.-, 358 pp.; ISBN 3-11(0)19.54-4.

Baile: Charles-Jumes: Variation in the Data: Can Linguistics Fver Become a Science? Kcááu, Hawaii. USA 1992. 25() pp.: \$ 15.()(): ISBN 1-8813(0)-(03-7.

Bell-Berti, Fredericka \& Raphael, Lawrence J. (eeds.) Producing Speech: Contemporary Issues for Katherine Salford Harris (= AIP Series in Modem Acoustics and Signal Processing) American Institute of Physics Press, New York 1995. 567 pp.: \$ 59.(0) (cloth); ISBN 1-56.396-286-1.

Breinung. Hors: Phonetik - Intonation - Kommunikation. 2 Standpunktc zur Sprach- und Kulturvermittlung. (jocthe Institut. München 1994. 224 pp; ISIBN 3-9.3()22()-()I-6.
Clark. J. \& Yallop, C.: An Introduction to Phonetics \& Phonology. Basil Blackwell, Oxford 1990). $X I 1+40()$ pp.: $£$ 14.95. ISBN ()-631-16182-1.

Cooke, Martin. Bees, Steve: Visual Representations of Speech Signals. Wilcy Professional Computing. John Wiley. Chichester 1993, X+386 pp.: $£$ 34.95. ISIBN ()- $\angle 71-93537-9$.

De Séchelles, Sitanne: I'articulation et la parole. Collection d'orthophonic. Masson. Paris 1993. $V I+1() 2$ pp.: FF II().-. ISBN 2-225-84I(0)-4.

Eissenberg. Peter. Ramers, Karl Heine \& Vater, Heinz, (Hrag.): Silbenphonologie des Deutschen (= Studien zur deutschen Grammatik 42). Gunther Narr Verlag. Tüibingen 1992. XIV. 329 S.. DIM 96.-. ISBN 3$8233-474.3-8$.

Gronnum. Nincl: The (iroundworks of Danish Intonation. An Introduction. Muscum Tusculanum Press. Copenhagen 1992. 11() pp.: DKK 178.(0). ISBN 877289-169-6. 
Hardcastle. W. J. \& Marchal. A. leds.): Speech Production and Speech Modelling. Proceedings of the NAT() Advanced Study Institute. Bonas. France. July 17-29, 1989 (= NATO Advanced Study Institutes Series D: Bchavioural and Social Sciences 5.5). Kluwer Academic Publ.. Dordrecht 1990. 460) pp.. Df1. 22()/ \& 79.(0). hardbound. ISBN ()-7923-()746-1.

Hess. Wolfgeng \& Sendlmeier: Walser IV (Hrsg.): Beiträge zur angewandten und experimentellen Phonetik (= \%eitschrift für Dialektologie und L.inguistik. Beiheft 72). Fran\% Steiner Verlag. Stuttgart 1992. 244 S.. DM 88,-. ISBN 3-515-()6(05()-2.

Jassem. Wikı): Exercises in English Pronunciation. Wydawnictwo Naukowe PWN, Warszalual 1995. 295 pp.: ISBN 8.3-(0)-11625-().

Laver: John: The Gift of Speech. Edinburgh Univ. Press. Edinburgh 1992. XVIII+4()() pp.: £ 45.-. ISBN (). $7486-(0) 313-1$.

Moosmialler: Silvic: Hochsprache und Dialekt in Österreich. Soziophonologische Untersuchungen \%u ihrer Abgrenzung in Wien, Graz. Salzburg. Innsbruck. Honlau Verlags (ies.. Wien 1991. 212 pp., DM 48.-. oS 336.-. ISBN 3-20.5-(054.55-5.

Ode, Cecilia \& Van Hewen. Vincent J. leds. ): Iixperimental Studies of Indonesian Prosody. (= Semaian 9). Vakgroep Talen en Culturen van Zuidoost-Azië en Oceanië, Rijksuniversitet le Leiden. 1994; 214 pp. + VII. I)f1. 4().(0). ISBN 9()-73()84-10)-5.

Parukhin. R.: Oral Spech. Controversies and Perspectives. Centrum Informacji, Poland 1993. 48 pp.; ISBN 83-9()1231-()-X.

Pompino-Marschall, B: Dic Silbenprosodic (I inguistische Arbeiten 247). Niemeyer, Tübingen 199(): 27() pp.. kart. D)M II(),-, ISB.N 3-484-30247-X.

Rienveld. Toni \& Van Hour. Roelend: Statistical Techniques for the Study of I anguage and I anguage Behavior. De Gruyter. Berlin 1993. XI+40) pp.: D)M 58.-. ISBN 3-11-(0136635-().
Saban. R.: Aux sources du langage articulé. Collection préhistoire. Masson. Paris 1993. 26.3 pp.: FF 178.-. ISBN 2-225-84166-7.

Sendlmeier; Walter F.: Sprachverarbeitung bei pathologischem (ichör. Georg Thieme Verlag. Stuttgart 1992. 224 pp.: DM 48.-. ISBN 3-1.3-7932()।-7.

Sorijiinvi. Annti \& Aulculko. Reijo: Synthesis of Certain Spech Sounds and Sentence Intonation of Hungarian, Finnish, and Finland-Swedish. (= Commentationes Humanarum Littcrarum 101). Socictas Scientiarum Fennica. Helsinki 1994: 88 S. ISBN $951 \cdot 653 \cdot 271 \cdot 3$.

Srengelhofen. Jackie (Ed.): Cleft Palate. The Nalture and Remediation of Communication Problems. Churchill I.ivingstone. Edinburgh. 1988. XI+194 pp.: £ 17.5(). ISBN ()-44.3-().3869-4.

Sundherg. Johem (Ed.): Gluing Tones Grouping in Music Composition, Performance and Listening. (= Publications 72). Royal Swedish Academy of Music. Stockholm 1992. 1()8 pp. + diskette. ISBN 918.5428-73-6.

Tench. P(ull: The Roles of Intonation in English Discourse. (= Forum L.inguisticum 31). Frankfurt/M. Bern. New York. Paris, Peter Lang 1990). ph. 95,(0) DM. ISBN 3-6.3I-426.54-2.

Uhmann, Suscmme: Fokusphonologie. Eine Analyse deutscher Intonationskonturen im Rahmen der nichtlinearen Phonologie (= Linguistische Arbeiten 252). Max Niemeyer Verlag, Tübingen 1991, 311 S.. kart. DM 124,()i). ISBN 3-484-3()2.52-6.

Vieregge. Wilhelm H. \& Gäschel. Joachim (Hrsg.): Gerold Lngeheuer. Phonctik und angrenzende (icbiete. Miszellaneen. Fragmente, Aufzeichnungen. Aus dem Nachlaß3 (= ZDL Beihefic 79). Franz. Stciner Vorlag, Stutgart 1993, 214 S., kart. I).M 76,-, ISBN 3-515-06296-3. 